\title{
Unusual 2001 periastron passage in the colliding-wind binary WR 140 (WC7pd+O4-5)
}

\author{
Sergey V. Marchenko ${ }^{1}$, Anthony F.J. Moffat ${ }^{2}$, Kiril P. Panov ${ }^{3}$, \\ Wilhelm Seggewiss ${ }^{4}$, and Victor G. Zubko ${ }^{5}$ \\ ${ }^{1}$ Department of Physics and Astronomy, Western Kentucky University, \\ 1 Big Red Way, Bowling Green, KY 42101-35\%6, USA \\ ${ }^{2}$ Département de physique, Université de Montréal, \\ C.P. 6128, Succ. Centre-Ville, QC H3C 3J7, Canada \\ ${ }^{3}$ Institute of Astronomy, Bulgarian Academy of Sciences, Sofia, Bulgaria \\ ${ }^{4}$ Sternwarte der Universität Bonn, Auf dem Hügel, D-53121 Bonn, BRD \\ ${ }^{5}$ NASA Goddard Space Flight Center, Greenbelt, MD 20771, USA
}

\begin{abstract}
The massive, long-period and highly-eccentric $(P=2899 \mathrm{~d}, e=0.88)$, colliding-wind binary WR 140 (WC7pd+O4-5) may be regarded as a clock-work for its predictable, repeatable IR outbursts related to dust formation right after each periastron passage. However, the 2001 periastron passage broke this monotonic trend. Our $U B V$ photometry, completely covering the past nine years, shows a series of quasi-regular fadings starting $\sim 2$ months after periastron passage $(\phi \simeq 0.02-0.05)$. Preliminary analysis points to dust as a probable cause of the eclipse-like behavior of the system, while the oscillating pattern hints towards instabilities in the wind-wind collision zone. At the same time, contemporaneous spectroscopy shows nothing unusual aside of the strong, but short-lived signs of wind-wind interaction $(\phi \simeq 0.99-1.02)$.
\end{abstract}

WR 140 (WC7pd+O4-5) is the best-studied long-period colliding-wind binary (see Setia Gunawan et al. 2001). At periastron passages, the extremely high eccentricity helps to create conditions favorable for rapid growth of dust cloud(s) presumably emerging from the wind-wind collision (WWC) zone.

Our two-site $U B V$ photometry (Figure 1) and multi-site high s/N spectroscopy completely covers two periastron passages. It reveals relatively rapid, phase-locked spectral variability at periastron, $\phi \simeq 0$, in the form of an extra emission component seen only in the low-ionization lines, C III and He I. In photometry, the two last periastron passages are remarkably different. The 2001 passage shows quasi-cyclic variability at $\phi \simeq 0.02-0.05$ (Figure 1).

Rapid growth of the extra emission components, along with their phasedependent blue-to-red shifts, allows one to link the emissions to the WWC zone and to conclude that the shocked gas can be treated in the radiative limit. The rapid cooling definitely helps to create dust. Change of the $U B V$ colors during the 'eclipses' shows the overall reddening of the star, which may be interpreted as coming from obscuration by moving dust clouds of the kind reported by Veen $e t$ al. (1998) and Kato et al. 2002ab. The typical size is $r \lesssim 2 \times 10^{13} \mathrm{~cm} \approx 300 \mathrm{R}_{\odot} \approx a$ 


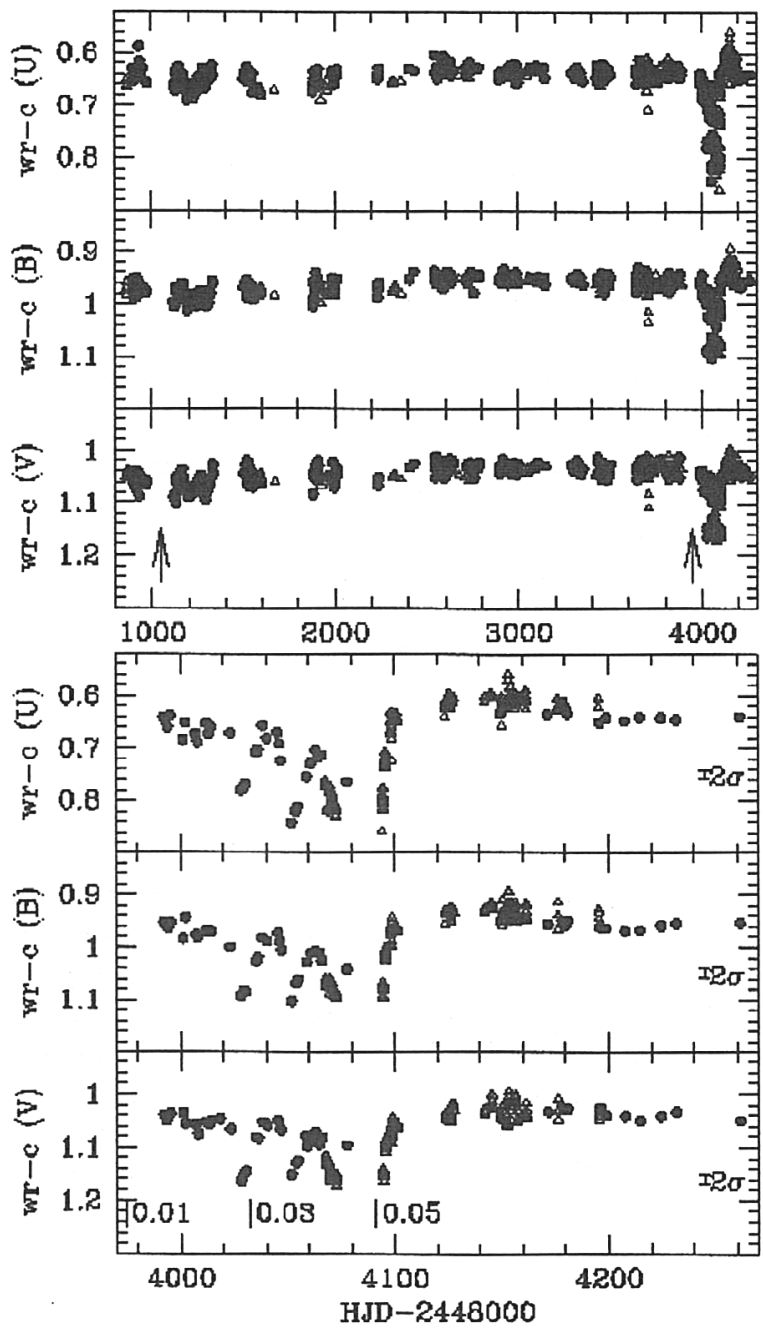

Figure 1. 2-site $U B V$ photometry of WR 140. Filled circles: APT in Arizona, USA. Open triangles: NAO Rozhen, Bulgaria. Arrows mark the 1993 and 2001 periastron passages. Phases are plotted in the lowermost section.

(the orbital separation at periastron). Cyclic behavior can be related to largescale instabilities in the WWC zone.

\section{References}

Kato, T., Haseda, K., Yamaoka, H., Takamizawa, K. 2002a, PASJ (Letters) 54, L51

Kato, T., Haseda, K., Takamizawa, K., Yamaoka, H. 2002b, A\&A (Letters) 393, L69

Setia Gunawan, D.Y.A., van der Hucht, K.A., Williams, P.M., Henrichs, H.F., Kaper, L., Stickland, D.J., Wamsteker, W. 2001, A\&A 376, 460

Veen, P.M., van Genderen, A.M., van der Hucht, K.A., Li, A., Sterken, C., Dominik, C. $1998, A \& A 329,199$ 